\title{
AN OPTIMAL RoUTE DISCOVERY USING BIOGEOGRAPHY BASED OPTIMIZATION FOR VEHICULAR AD HOC NETWORKS
}

\author{
Shaeista Begum ${ }^{1}$, Nagaraj B. Patil ${ }^{2}$ and Vishwanath $\mathrm{P}^{3}$ \\ ${ }^{1}$ Department of Computer Science and Engineering, \\ Government Engineering College, Raichur, India \\ ${ }^{2}$ Department of Computer Science and Engineering, \\ Government Engineering College, Gangavathi, India \\ ${ }^{3}$ Department of Electronics and Communication Engineering, \\ H.K.E.S's S.L.N. College Of Engineering, Raichur, India
}

\begin{abstract}
Vehicular ad hoc network (VANET) is a subdivision of the mobile ad hoc networks which uses the moving vehicles as mobile nodes to form the mobile network. In conventional vehicular communications, the restricted radio frequency bandwidth affects the network performances. Therefore, Visible Light Communication (VLC) is integrated with the growing vehicular ad hoc network to obtain high data rate and less energy consumption during the communication. In this paper, vehicular communication is integrated with visible light communication to avoid the issues caused by the restricted radio frequency bandwidth. Moreover, the Routing using Biogeography Based Optimization (RBBO) is proposed to develop an optimal route between the source vehicles to the destination. This research performs two different communications such as vehicle to vehicle and vehicle to the infrastructure. The performance of the RBBOVLC-VANET method is analyzed by means of throughput, packet delivery ratio, delay and routing overhead as well as these performances are compared with the existing method namely ant colony optimization based routing protocol. The throughput of the routing using the biogeography based optimization method is 589.763 kbps for 500 nodes which is high when compared to the existing method.
\end{abstract}

\section{KEYWORDS}

Biogeography Based Optimization, Delay, Optimal Route, Throughput, Vehicular Ad Hoc Network, Visible Light Communication.

\section{INTRODUCTION}

VANET is one of the special types of mobile ad hoc networks (MANETs) which allows vehicles to generate the self-organizing mobile wireless network without any pre-existing infrastructure [1] [2] [3]. The vehicles of the VANET are act as a node or router which is used to exchange the data among the access points and vehicles [4]. The main goal of the VANET is to develop an Intelligent Transport System (ITS) to provide numerous general applications such as vehicle safety, collision avoidance, traffic regulation and protecting the blind while crossing the roads [5] [6]. The VANET has two components such as vehicle and the roadside infrastructures. Since, the communication between the vehicles are named as vehicle to vehicle (V2V) communication whereas the communication among the vehicle and roadside infrastructure is called as Vehicle to Infrastructure (V2I) communication [7] [8]. The nodes of the VANET are equipped with On- 
Board Unit $(\mathrm{OBU})$ at a standard position for supporting the aforementioned V2V and V2I communications [9] [10].

The routing between the vehicular connections is difficult due to the vehicles movement and frequent changes in the network topology. The network expansion over the huge scale causes the higher routing overheads for the routing protocols [11] [12]. Moreover, the data transmission through the VANET is affected by different constraints such as non-uniformity of vehicle density and intermittent connection [13]. Conventionally, the VANET uses the Radio Frequency (RF) for supporting the communication among the vehicular communications. In RF-based VANET, the efficiency of the network is affected, when the interference avoidance cost is increased with respect to the vehicle density. Additionally, the network performance is restrained based on the restricted RF bandwidth. Therefore, the VLC is utilized in the VANET for supporting the VANET communications due to its different advantages of high spatial reuse ratio, wide freelicensed spectrum and higher power efficiency [14]. The VLC provides reliable and energyefficient data transmission compared to the RF. However, the network is affected by the unequal load distribution through the network and data heterogeneity [15]. The major contributions of this research paper are given as follows:

- The VANET is integrated with the VLC for avoiding the multiple distortions while transmitting the data packets.

- The Routing using Biogeography Based Optimization (RBBO) is proposed to generate the optimal route between the source to the destination. This route generation is optimized by considering the multiple objectives such as residual energy, distance and number of hops.

- The proposed RBBO-VLC-VANET is used to obtain two different communications such as V2V and V2I and also it minimizes the delay and increases the throughput over the VLC-VANET.

The major contributions of this paper are given as follows: The literature survey about the existing routing methods over the VANET is given in the section 2 . The problems found from the literature survey and solution to overcome that problem is described in the section 3 . The route generation using the biogeography-based optimization over the VLC-VANET is explained in the section 4. The results and discussion of the RBBO-VLC-VANET is given in the section 5. Finally, the conclusion is made in section 6.

\section{LITERATURE SURVEY}

Chinnasamy et al. [16] presented the Wagon Next Point Routing Protocol (WNPRP) to generate a continuous and reliable data transmission between the vehicles. The Hello Message was frequently transmitted between the adjacent wagons (i.e., vehicles) to obtain significant information about adjacent vehicles. There are three different information was obtained using Hello Message transmission such as direction, location and speed of the vehicle. This collected information was used in the WNPRP to maintain the connectivity and constant stability among the source and destination. The developed WNPRP was analyzed only for lesser number of nodes. For an effective VANET communication, the network area should be analyzed with a higher number of vehicles.

Aravindhan and Dhas [17] developed the hybrid clustering and destination-aware routing protocol to obtain an optimal cluster head and route between the vehicles. In hybrid clustering, the clusters were formed by integrating the geographic and context-based clustering method. Next, the inter-clustering routing was obtained using the destination-aware routing protocol. The 
direction and destination of cluster heads were considered during the optimal cluster head selection as well as this cluster head was used to generate the optimal route. Therefore, the developed clustering and routing algorithm was used to minimize the traffic and delay in the VANET communication. The amount of control message transmission was very high with respect to increase the number of clusters.

Srivastava et al. [18] presented the Adaptive Intersection Selection Mechanism (ASIM) using Ant Colony Optimization (ACO) to generate the routing path based on the constraints of the multiple quality of service. This ASIM was followed two strategies: At first, the real time road evaluation was obtained based in the prediction-based mechanism. Next, the route among the two adjacent vehicles was identified instead of detecting the longer route between the nodes. The higher data transmission and reduction in unwanted path identification were obtained using this ASIM. The priority of the candidate route selection was considered only the delay and connectivity, it failed to consider the energy in the routing process.

Masini et al. [19] developed the applications of vehicular crowd sensing, where the vehicles were utilized to gather the urban data required by the data centers. The vehicles in the network were considered as generating the data packets which contains the information about the vehicles. Here, both the VLC and IEEE 802.11p were integrated and also it was used individually during the communication. The multi-hop communication was initialized, when there is no possible intermediate connection between the vehicles. The number of packets delivered to the Road Side Unit (RSU) was affected due to the high directivity between the transmitter and receiver.

Gawas and Govekar [20] presented the Selective Cross-Layer design-based Ant Colony Optimized Ad-Hoc On Demand Distance Vector (SCL-ACO-AODV) routing algorithm was developed to generate the route through the VANET. The difficulty during the route generation was avoided by ACO and allocation of slot was obtained using the Particle Swarm Optimization (PSO). The AODV was used to initialize the data transmission between the source and destination. The redundant traffic congestion and confusion were minimized using this SCLACO-AODV method. Moreover, the performance of the SCL-ACO-AODV was mainly based in the selected intermediate nodes and candidate routing paths as well as the selection of intermediate node considers only the distance and energy of the nodes.

\section{Problem statement}

The problems found from the existing researches and solution to solve that problem using RBBO-VLC-VANET is described in this section.

The inappropriate fitness function selection during the route generation causes the packet loss through the network. The route selection through the ASIM [18] considers only the delay and connectivity for data transmission. This ASIM doesn't consider the energy of the nodes, because of this the node with limited battery power affects the number of packets received in the destination. Moreover, the SCL-ACO-AODV [20] considers only the distance and energy during the candidate path selection. The amount of packets received at the RSU is minimized due to the reduced connectivity in the VLC communication [19].

\section{Solution:}

In this RBBO-VLC-VANET method, the Biogeography Based Optimization (BBO) is used to generate the routing path for transmitting the data packets between the source vehicle to the RSU. The multiple objective values considered to generate the optimal route are residual energy, 
distance and number of hops. This helps to increase the number of packets received by the destination. Moreover, the shortest path generation using the RBBO minimizes the delay while transmitting the data packets.

\section{RBBO-VLC-VANET METHOD}

In this RBBO-VLC-VANET, an optimal path between the source vehicle and the RSU is obtained by using the BBO algorithm. This BBO is optimized by using the residual energy, distance and number of hops. Here, the data transmission between the vehicles is carried out through the VLC channel. This VLC data transmission helps to avoid the multiple distortions during the data transmission. This RBBO-VLC-VANET transmits the data between the vehicles and vehicle to infrastructure namely V2V and V2I. Moreover, this RBBO-VLC-VANET is used to transmit the high amount of data packets with less packet loss. The flowchart of the RBBOVLC-VANET method is shown in the Figure 1.

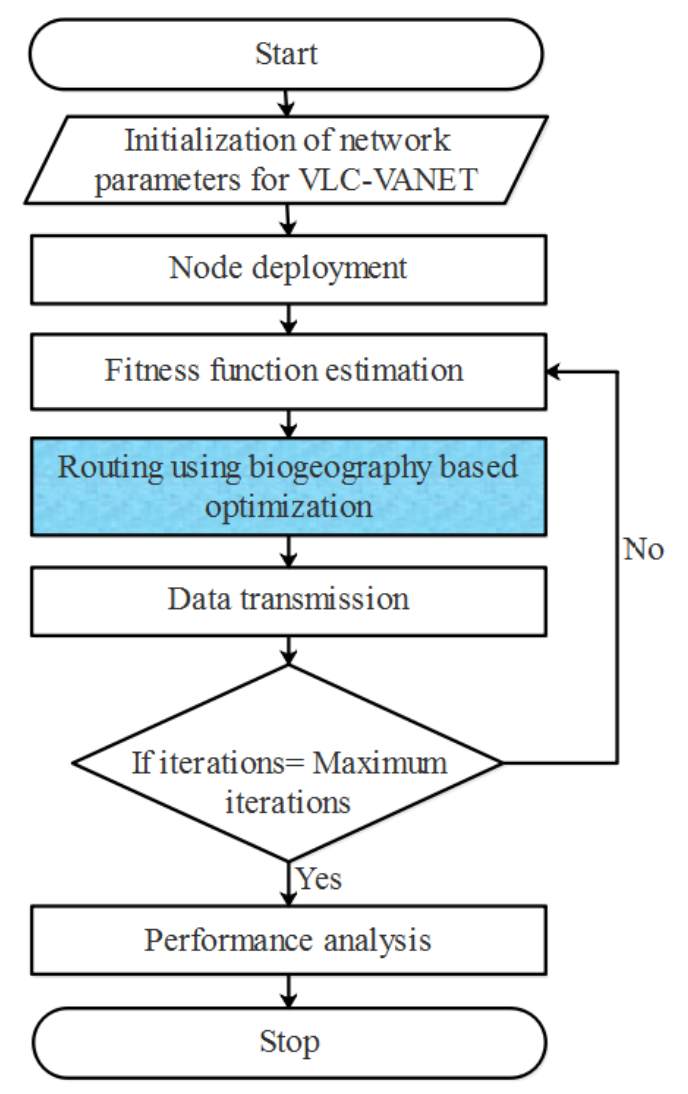

Figure 1. Flowchart of the RBBO-VLC-VANET method

\subsection{VLC-VANET system model}

The VLC-VANET system model is comprised of vehicles as mobile nodes and lighting sources as transmitting/receiving sources. In this VLC-VANET, both the lighting infrastructure and mobile nodes are equipped with the transmitter and receiver to transmit and receive the information from the adjacent vehicles. For example, the VLC transmitter and receiver are equipped with the headlights and brake lights of a vehicle. The VLC transmitter and VLC receiver are connected through the free space optical communication channel. The main requirement of the VLC is the Line-of-Sight $(\mathrm{LoS})$ especially in outdoor vehicle communications. 
The light emitted from the transmitter contains the data packets which are transferred through the wireless medium. Here, the direct LoS is used as communication technology between the vehicles which used to minimize the multipath distortion during data transmission. The channel model of this VLC-VANET is expressed in the equation (1).

$$
H_{\text {Los }}=\left\{\begin{array}{cc}
\frac{(B+1) A}{2 \pi D i s t^{2}} \cos ^{b}(\varphi) T(\psi) g(\psi) \cos (\psi) & 0 \leq \psi \leq \psi_{c} \\
0 & \psi>\psi_{c}
\end{array}\right.
$$

Where, $b$ is the order of Lambertian emission; $A$ is the detector area; distance between one vehicle to other vehicle is Dist; optical band-pass filter of transmission is $T(\psi)$; concentrator gain is $g(\psi)$, receiver's field of view is $\psi_{c}$; angle of impedance is $\psi$ and angle of irradiance is $\varphi$.

\subsection{Identification of routing path using BBO}

The location of nodes deployed in the VLC-VANET is given as input to the BBO algorithm for generating the route between the source vehicle to the destination vehicle. The multiple objectives considered in this RBBO-VLC-VANET method are residual energy, distance and number of $\mathrm{BS}$. This section provides an overview of $\mathrm{BBO}$ and route generation using $\mathrm{BBO}$.

\subsubsection{Overview of biogeography based optimization}

Initially, the BBO is developed by Dan Simon in 2008 [21]. BBO is generally inspired by the geographical assignment of biological species and the Habitat Suitability Index (HSI) is used to specify each geographical zone of BBO. An extra index utilized to mention the habitat area and conditions of livelihood is specified as Suitability Index Variable (SIV). The HSI value and amount of species is equal to the habitat's fitness value. The features from the solution of higher HSI are accepted for enhancing lower HSI solution. The immigration and emigration rate of the habitat are $\lambda$ and $\mu$ are used to specify the single species model. This immigration and emigration rate is shown in the equation (2) and (3) respectively.

$$
\lambda=I\left(1-\frac{k}{n_{s}}\right)
$$

Where, the maximum immigration rate is represented as $I$; number of species in the habitat is represented as $k$ and maximum amount of species in the habitat is $n_{s}$.

$$
\mu=\frac{E \times k}{n_{s}}
$$

Where, $E$ represents the maximum emigration rate. The processes of the $\mathrm{BBO}$ algorithm are given as follows:

\subsubsection{Migration}

Consider the candidate solutions and the optimization problem for the BBO initialization, where each solution specified using the dimension vector $(n)$ is called as habitat. Let, the habitat's 
dimension in each dimension is SIV as well as the habitat's goodness is equal to the amount of species and HSI value. The low HSI solution shares the information with higher HSI solution to improve the solution obtained during the searching process. The transmission of HSI solution depends on the immigration and emigration rate and the two different habitats are selected from the $\mathrm{BBO}$ population. Initially, one habitat $\left(H_{i}\right)$ is selected by considering the immigration rate $\left(\lambda_{i}\right)$ and one more habitat $\left(H_{j}\right)$ is selected by considering the emigration rate $\left(\mu_{j}\right)$. Subsequently, the random selection of SIVs are transferred from $\left(H_{j}\right)$ and appears in $\left(H_{i}\right)$.

\subsubsection{Mutation}

Generally, the rapid changes in habitat and deviation from the equilibrium location are occurred due to natural disasters caused in the geographical region. Consequently, the same effect is illustrated in the BBO using the operation of mutation. This mutation operation is accomplished using the number of species in each habitat, which is shown in equation (4) and (5). The value of probability is allocated for each habitat to perform the mutation operation. The possibility for mutation is less and a solution is closer to the optimal solution, when the probability value is high in the BBO. However, the possibility of mutation is high and a solution is far from the optimal solution, when the probability value is low.

$$
P_{S}^{h}=\left\{\begin{array}{cc}
-\left(\lambda_{s}+\mu_{s}\right) P_{s}+\mu_{s+1} P_{s+1}, & s=0 \\
-\left(\lambda_{s}+\mu_{s}\right) P_{s}+\lambda_{s-1} P_{s-1}+\mu_{s+1} P_{s+1} & 1<s \leq s_{\max }-1 \\
-\left(\lambda_{s}+\mu_{s}\right) P_{s}+\lambda_{s-1} P_{s-1} & s=s_{\max } \\
m(s)=m_{\max }\left(\frac{1-P_{s}}{P_{\max }}\right) &
\end{array}\right.
$$

Where, the higher amount of species in the BBO is $s_{\max }$; mutation rate of $s$ species is $s_{\max }$; maximum mutation rate and probability are represented as $m_{\max }$ and $P_{\max }$ respectively.

\subsubsection{Optimal path generation using BBO}

This BBO is used to generate the optimal route to achieve a high amount of packets received at the destination RSU. The issues related to frequent changes in network topology and high density of vehicles are overcome by generating the optimal path and transmitting the data through the VLC channel.

\subsubsection{Representation and initialization of BBO}

The habitats of the BBO are specified by the possible paths from the source vehicle to the RSU. Moreover, the dimension of the habitat is identical to its number of intermediate vehicles in the routing path. Consider, the habitat $i$ is $H_{i}=\left(H_{i, 1}(t), H_{i, 2}(t), \ldots H_{i, m}(t)\right)$, whereas the next hop vehicle is specified as $H_{i, v}, 1 \leq v \leq m$.

\subsubsection{Fitness function formulation for $B B O$}

In this RBBO-VLC-VANET method, three different objective values such as residual energy, distance between one vehicle node to another vehicle node and number of hops connected to each vehicle node. 


\section{a. Residual energy}

The residual energy $(R E)$ of each node is considered as a significant fitness value, because it shows the amount of energy exist in each node. This used to select only the node which has higher residual energy while transmitting the data packets. Because, the node with limited energy creates node/link failure during data transmission which causes the packet loss. The following equation (6) shows the residual energy.

$$
R E=\sum_{i=1}^{m} E_{H_{i}}
$$

Where, $m$ represents the number of vehicles in the routing path and $E_{H_{i}}$ represents the residual energy of the $i^{\text {th }}$ vehicle in the habitat $H$.

\section{b. Distance}

The distance (Dist) between the one vehicle to another vehicle is considered to select the shortest path between the source vehicle to destination RSU. Moreover, this shortest path identification is used to minimize the delay during communication. Equation (7) expresses the distance between the vehicles.

$$
\text { Dist }=\sum_{i=1}^{m} \operatorname{dis}\left(H_{i}, D V\right)
$$

Where, destination vehicle is represented as $D V$ as well as distance between the $i^{\text {th }}$ vehicle in the habitat $H$ and $D V$ is represented as $\operatorname{dis}\left(H_{i}, D V\right)$.

\section{c. Number of hops}

The amount of Next Hop Vehicle (NHV) to the respective node defines the number of hops $(\mathrm{NH})$. The number of hops is required to be less, because it causes higher energy consumption in VANET.

$$
N H=\sum_{i=1}^{m} M_{H_{i}}
$$

Where, $M_{H_{i}}$ represents the amount of nodes connected in the path.

In this fitness function derivation, the multiple objectives are converted into single objective by assigning weighted value to each objective value. Equation (9) shows the HSI/fitness value of the BBO (5).

$$
H S I=\alpha_{1} \times R E+\alpha_{2} \times \text { Dist }+\alpha_{3} \times N H
$$

Where, the $\alpha_{1}, \alpha_{2}$ and $\alpha_{3}$ represents the weighted values used in the fitness function. The calculated values of HSI are used to update the immigration and emigration rate of each habitat. 


\subsubsection{Migration and mutation}

The immigration and emigration rate are used to select two different habitats during the migration process. Next, the NHV from the higher HSI solution has appeared in lower HSI solution. Here, one location is randomly created among the 1 and $m^{\text {th }}$ dimension to accomplish the migration process. From the generated location, all NHV from the $H_{j}$ appears in $H_{i}$. Therefore, the habitats are updated until the optimal solution is obtained in the BBO. The habitat $H_{i}$ considered using the mutation probability. The selection of habitat is identified using the emigration and immigration rate. The probability of selecting the habitat is less, when the mutation probability is high during mutation process. Otherwise, the probability of selecting the habitat is high, when the mutation probability is less during mutation process. In habitat $H_{i}$, the randomly selected location changes its NHV by selecting the random NHV within its transmission range.

\section{RESULTS AND DISCUSSION}

The results and discussion of the proposed RBBO-VLC-VANET are described in this section. The implementation and simulation of the RBBO-VLC-VANET is carried out in the MATLAB R2018a which is operated in the in a Windows 8 operating system with an Intel core i3 processor and 4GB RAM. In this RBBO-VLC-VANET, the VLC is used as a communication channel for supporting the data transmission between the vehicles. The routing path between the source vehicle to the RSU is generated by using the BBO. The number of vehicles deployed in the network area are varied as 50,100, 200 and 500 which are deployed in the area of $500 \times 500 \mathrm{~m}^{2}$. The specification parameters of this RBBO-VLC-VANET method are given in Table 1.

Table 1. Specification parameters

\begin{tabular}{|l|l|}
\hline Parameter & Value \\
\hline Area & $500 \times 500 \mathrm{~m}^{2}$ \\
\hline Number of vehicles & $50,100,200$ and 500 \\
\hline RSU location & Center of the network area \\
\hline Transmission range & $250 \mathrm{~m}$ \\
\hline Communication channel & VLC \\
\hline Packet size & 4000 bits \\
\hline Routing protocol & BBO \\
\hline
\end{tabular}

\subsection{Performance analysis}

The performance of the RBBO-VLC-VANET is analyzed in terms of the Throughput, Packet Delivery Ratio (PDR), delay and routing overhead. Next, the RBBO-VLC-VANET is evaluated with SCL-ACO-AODV [20] to show the efficiency of RBBO-VLC-VANET. The performance analysis is described as follows:

\subsubsection{Throughput}

Throughput is defined as a number of packets successfully received at the RSU during the simulation rounds. Generally, throughput is measured as kilobits per second or Megabits per second. 


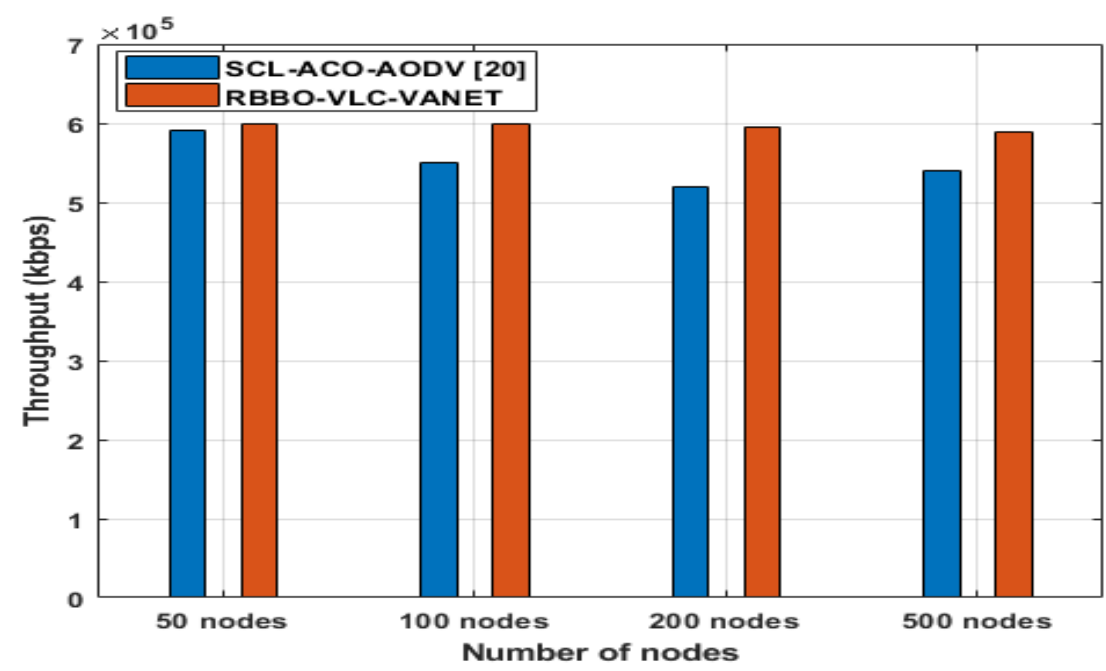

Figure 2. Comparison of throughput for varying nodes

The throughput comparison of the RBBO-VLC-VANET with SCL-ACO-AODV [20] is shown in the Figure 2. Figure 2 shows that the RBBO-VLC-VANET method obtains higher throughput than the SCL-ACO-AODV [20]. For example, the throughput of the RBBO-VLC-VANET method is $589.763 \mathrm{kbps}$ for 500 nodes which is high when compared to the SCL-ACO-AODV [20] method's throughput i.e., $540 \mathrm{kbps}$. The elimination of multiple distortions using VLC and optimal path generation using BBO are used to achieve the higher throughput in the RBBO-VLCVANET method.

\subsubsection{Packet Delivery Ratio}

PDR is the ratio between the Number of packets successfully received at the RSU to the total packets generate at the source vehicle. Expression for PDR is shown in the equation (10).

$$
P D R=\frac{\text { Amount of Packets sucessfully received at the RSU }}{\text { Total packets transmitted }}
$$

Figure 3 shows the comparison of the PDR for the RBBO-VLC-VANET with SCL-ACO-AODV [20]. This comparison shows that the RBBO-VLC-VANET method has higher PDR than the SCL-ACO-AODV [20]. For example, the PDR of the RBBO-VLC-VANET method is $98.998 \%$ which is high when compared to the SCL-ACO-AODV [20]. The integration of VLC into the VANET is used to increase the number of packets received by the RSU. Moreover, the SCLACO-AODV [20] doesn't consider the appropriate fitness function value which leads to cause the packet loss during route generation. 


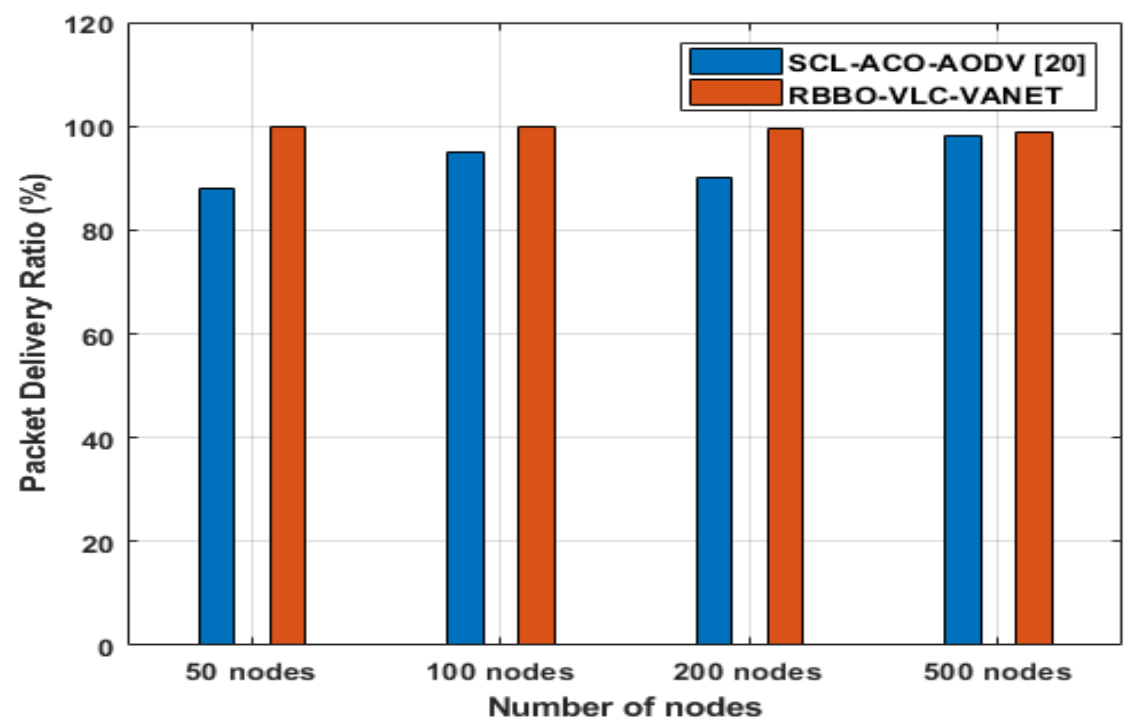

Figure 3. Comparison of PDR for varying nodes

\subsubsection{Packets' delay}

Delay is the total required to transmit the data packet from the source to the destination which is expressed in the equation (11).

$$
\text { Packets' Delay }=\frac{\text { Total time required to transmit the data packets }}{\text { Amount of packets sucessfully received at the } R S U}
$$

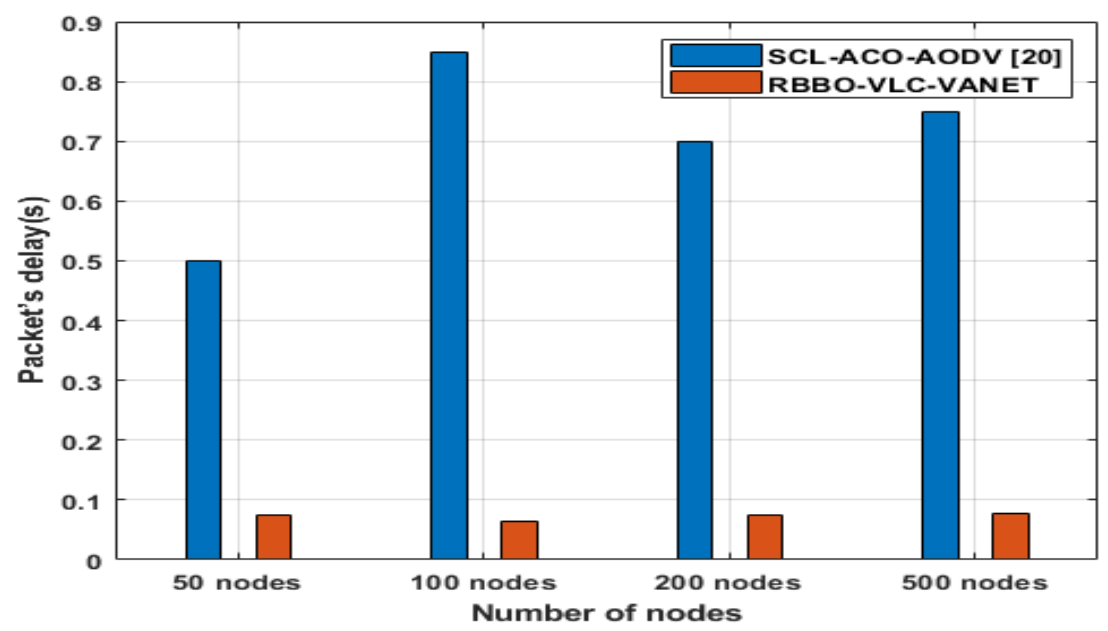

Figure 4. Comparison of delay for varying nodes

The delay comparison of the RBBO-VLC-VANET with SCL-ACO-AODV [20] is shown in the Figure 4. Figure 4 shows that the RBBO-VLC-VANET method achieves lesser delay than the SCL-ACO-AODV [20]. For example, the delay of the RBBO-VLC-VANET method is $0.0783 \mathrm{~s}$ for 500 nodes which is less when compared to the SCL-ACO-AODV [20] method's delay i.e., $0.75 \mathrm{~s}$. The delay during the data transmission is less by identifying the shortest path between the 
International Journal of Computer Networks \& Communications (IJCNC) Vol.13, No.1, January 2021

source vehicle to the destination. But, the SCL-ACO-AODV [20] has a higher delay because of the traffic that occurred in the VANET.

\subsubsection{Routing overhead}

Routing overhead is defined as the ratio between the number of control packets and amount of packets successfully received at the RSU. The routing overhead is expressed in the following equation (12).

$$
\text { Routing overhead }=\frac{\text { Amount of control packets }}{\text { Amount of packets sucessfully received atthe } R S U}
$$

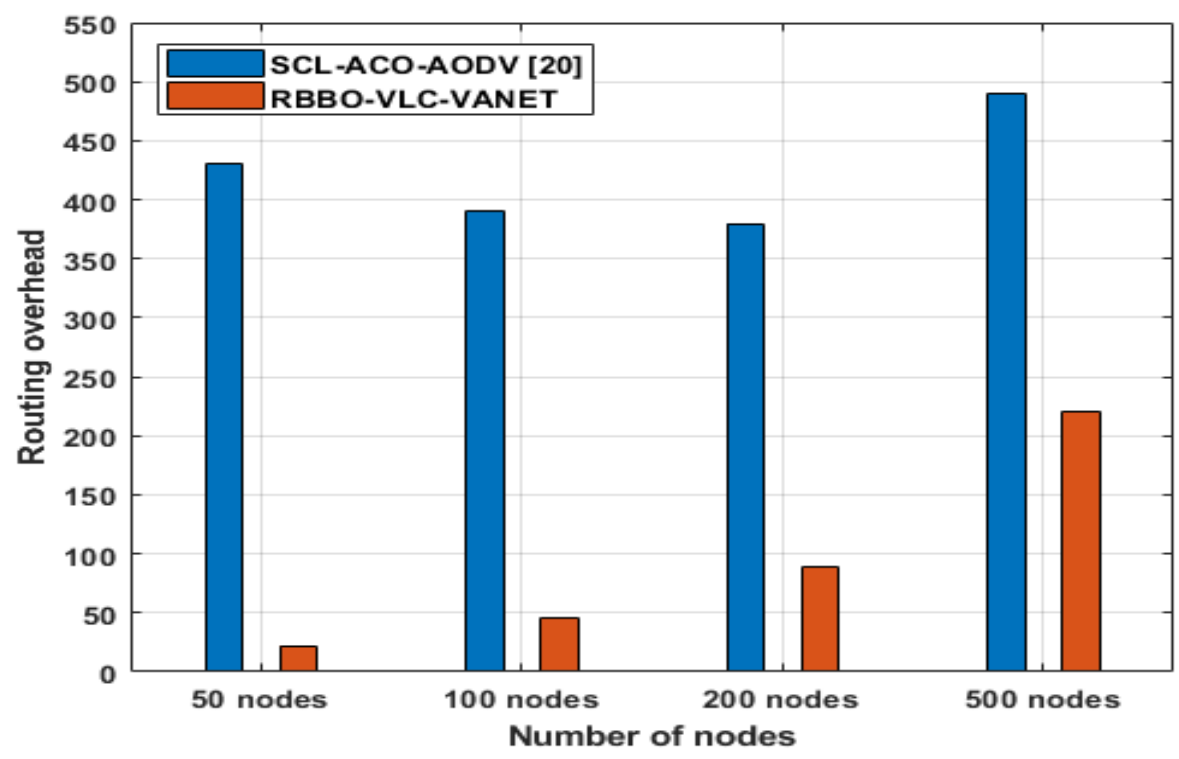

Figure 5. Comparison of routing overhead for varying nodes

Figure 5 shows the comparison of the routing overhead for the RBBO-VLC-VANET with SCLACO-AODV [20]. This comparison shows that the RBBO-VLC-VANET method has lesser routing overhead than the SCL-ACO-AODV [20]. For example, the routing overhead of the RBBO-VLC-VANET method is 220.2752 which is less when compared to the routing overhead of SCL-ACO-AODV [20] i.e., 490. The optimal route generation using BBO through the VLCVANET is used to minimize the routing overhead while transmitting the data packets.

Table 2. Comparative analysis of the RBBO-VLC-VANET with SCL-ACO-AODV

\begin{tabular}{|c|c|c|c|c|c|c|c|c|}
\hline \multirow{2}{*}{$\begin{array}{l}\text { Number } \\
\text { of nodes }\end{array}$} & \multicolumn{2}{|c|}{ Throughput (kbps) } & \multicolumn{2}{|c|}{ PDR (\%) } & \multicolumn{2}{|c|}{ Delay (s) } & \multicolumn{2}{|c|}{ Routing overhead } \\
\hline & $\begin{array}{l}\text { SCL- } \\
\text { ACO- } \\
\text { AODV } \\
{[20]}\end{array}$ & $\begin{array}{l}\text { RBBO- } \\
\text { VLC- } \\
\text { VANET }\end{array}$ & $\begin{array}{l}\text { SCL- } \\
\text { ACO- } \\
\text { AODV } \\
{[20]}\end{array}$ & $\begin{array}{l}\text { RBBO- } \\
\text { VLC- } \\
\text { VANET }\end{array}$ & $\begin{array}{l}\text { SCL- } \\
\text { ACO- } \\
\text { AODV } \\
{[20]}\end{array}$ & $\begin{array}{l}\text { RBBO- } \\
\text { VLC- } \\
\text { VANET }\end{array}$ & $\begin{array}{l}\text { SCL- } \\
\text { ACO- } \\
\text { AODV } \\
{[20]}\end{array}$ & $\begin{array}{l}\text { RBBO- } \\
\text { VLC- } \\
\text { VANET }\end{array}$ \\
\hline 50 & 590 & 598.939 & 88 & 99.821 & 0.5 & 0.075 & 430 & 22.423 \\
\hline 100 & 550 & 598.38 & 95 & 99.797 & 0.85 & 0.064 & 390 & 45.087 \\
\hline 200 & 520 & 595.335 & 90 & 99.594 & 0.7 & 0.074 & 380 & 88.208 \\
\hline 500 & 540 & 589.763 & 98 & 98.998 & 0.75 & 0.078 & 490 & 220.275 \\
\hline
\end{tabular}


Table 2 shows the comparative analysis of the RBBO-VLC-VANET with SCL-ACO-AODV [20] for a different numbers of nodes such as 50, 100, 200 and 500. From the Table 2, it shows that the performance of the RBBO-VLC-VANET is improved than the SCL-ACO-AODV [20]. The SCL-ACO-AODV [20] has lesser performance due to its inappropriate fitness function formulation during route selection. The integration of VLC with VANET is used to increase the number of packets received at the RSU. The multiple objectives such as residual energy, distance and number of hops are considered in the BBO to identify the optimal route between source vehicle to RSU. The packet loss during the data communication is minimized by avoiding the node failure in the routing path. Therefore, the number of packets received at the RSU is increased through the VANET. The distance considered in the fitness function helps to detect the shortest path which used to minimize the delay in VANET communication.

\section{CONClusion}

In this RBBO-VLC-VANET, the VLC is integrated with the VANET to achieve the higher throughput during communication. The $\mathrm{BBO}$ based route generation is proposed to obtain the optimal path between the source vehicle to the destination RSU. Here, the BBO is optimized by using the three different objective values such as residual energy, distance and number of hops. Therefore, this optimal path generation is used to overcome the issues related to frequent changes in network topology and node failure. This RBBO-VLC-VANET method is used to obtain the higher throughput and lesser delay by identifying the optimal path. Therefore, the proposed RBBO-VLC-VANET is operated in both the $4 \mathrm{G}$ and $5 \mathrm{G}$ networks. From the performance analysis, it knows that the RBBO-VLC-VANET method provides better performance than the existing SCL-ACO-AODV method. The throughput of the RBBO-VLC-VANET is $589.763 \mathrm{kbps}$ for 500 nodes which is high when compared to the throughput of SCL-ACO-AODV i.e., $540 \mathrm{kbps}$.

\section{CONFLICTS OF INTEREST}

The authors declare no conflict of interest.

\section{REFERENCES}

[1] Liu, H., Yang, L. \& Zhang, Y. (2015) "Improved AODV routing protocol based on restricted broadcasting by communication zones in large-scale VANET", Arabian Journal for Science and Engineering, Vol. 40, No. 3, pp857-872.

[2] Li, N., Martínez-Ortega, J.F., Díaz, V.H. \& Fernandez, J.A.S. (2018) "Probability prediction-based reliable and efficient opportunistic routing algorithm for VANETs", IEEE/ACM Transactions on Networking, Vol. 26, No. 4, pp.1933-1947.

[3] Al-Kharasani, N.M., Zukarnain, Z.A., Subramaniam, S.K. \& Hanapi, Z.M. (2020) "An Adaptive Relay Selection Scheme for Enhancing Network Stability in VANETs”, IEEE Access, Vol. 8, pp128757-128765.

[4] Suganthi, B. \& Ramamoorthy, P. (2020) "An Advanced Fitness Based Routing Protocol for Improving QoS in VANET", Wireless Personal Communications, pp1-23.

[5] Purkait, R. \& Tripathi, S. (2020) "Fuzzy logic based multi-criteria intelligent forward routing in VANET”, Wireless Personal Communications, Vol. 111, No. 3, pp1871-1897.

[6] Wagh, M.B. \& Gomathi, N. (2018) "Route discovery for vehicular ad hoc networks using modified lion algorithm", Alexandria engineering journal, Vol. 57, No. 4, pp3075-3087.

[7] Bello-Salau, H., Aibinu, A.M., Wang, Z., Onumanyi, A.J., Onwuka, E.N. \& Dukiya, J.J. (2019) “An optimized routing algorithm for vehicle ad-hoc networks", Engineering Science and Technology, an International Journal, Vol. 22, No. 3, pp754-766.

[8] Zhang, D., Zhang, T. \& Liu, X. (2019) "Novel self-adaptive routing service algorithm for application in VANET", Applied Intelligence, Vol. 49, No. 5, pp1866-1879. 
International Journal of Computer Networks \& Communications (IJCNC) Vol.13, No.1, January 2021

[9] Patil, S.D. \& Ragha, L. (2020) “Adaptive fuzzy-based message dissemination and micro-artificial bee colony algorithm optimised routing scheme for vehicular ad hoc network", IET Communications, Vol. 14, No. 6, pp.994-1004.

[10] Abuashour, A. \& Kadoch, M. (2017) "Performance improvement of cluster-based routing protocol in VANET", IEEE Access, Vol. 5, pp15354-15371.

[11] Moridi, E. \& Barati, H. (2017) "RMRPTS: a reliable multi-level routing protocol with tabu search in VANET", Telecommunication Systems, Vol. 65, No. 1, pp127-137.

[12] Chen, C., Liu, L., Qiu, T., Yang, K., Gong, F. \& Song, H. (2018) “Asgr: An artificial spider-webbased geographic routing in heterogeneous vehicular networks", IEEE Transactions on Intelligent Transportation Systems, Vol. 20, No. 5, pp1604-1620.

[13] Wu, J., Fang, M., Li, H. \& Li, X. (2020) "RSU-Assisted Traffic-Aware Routing Based on Reinforcement Learning for Urban Vanets", IEEE Access, Vol. 8, pp5733-5748.

[14] Chen, J. \& Wang, Z. (2019) "Topology Control in Hybrid VLC/RF Vehicular Ad-Hoc Network", IEEE Transactions on Wireless Communications, Vol. 19, No. 3, pp1965-1976.

[15] Msongaleli, D.L. \& Kucuk, K. (2019) "Optimal resource utilisation algorithm for visible light communication-based vehicular ad-hoc networks”, IET Intelligent Transport Systems, Vol. 14, No. 2 , pp65-72.

[16] Chinnasamy, A., Prakash, S. \& Selvakumari, P. (2016) "Wagon next point routing protocol (wnprp) in vanet “, Wireless Personal Communications, Vol. 90, No. 2, pp875-887.

[17] Aravindhan, K. \& Dhas, C.S.G. (2019) "Destination-aware context-based routing protocol with hybrid soft computing cluster algorithm for VANET”, Soft Computing, Vol. 23, No. 8, pp24992507.

[18] Srivastava, A., Prakash, A. \& Tripathi, R. (2020) “An adaptive intersection selection mechanism using ant Colony optimization for efficient data dissemination in urban VANET", Peer-to-Peer Networking and Applications, pp1-19.

[19] Masini, B.M., Bazzi, A. \& Zanella, A. (2018) "Vehicular visible light networks for urban mobile crowd sensing", Sensors, Vol. 18, No. 4, p1177.

[20] Gawas, M.A. \& Govekar, S.S. (2019) "A novel selective cross layer based routing scheme using ACO method for vehicular networks", Journal of Network and Computer Applications, Vol. 143, pp34-46.

[21] Verma, J. and Kesswani, N. (2018) "BIGM: A Biogeography Inspired Group Mobility Model for Mobile Ad Hoc Networks", International Journal of Wireless Information Networks, Vol. 25, No. 4, pp.488-505. 


\section{AUTHORS}

Shaeista Begum perceived her B.E from Bapuji Institute of Engineering and Technology, Davangere, Karnataka in 2005 and M.Tech from University of BDT College of Engineering, Davangere, Karnataka in 2007. From 2007 to 2008 she worked as a lecturer in GM Institute of Technology, Davangere. From 2008 to 2010 worked as a lecturer in BKIT, Bhalki, Karnataka. From 2010 to 2011 worked as a lecturer in Women's Government Polytechnic, Gulbarga. Presently working as an Assistant Professor in Government Engineering College, Raichur since 2011. Her research area is Computer Network.

Nagaraj B. Patil received his B.E. degree from the Gulbarga University Gulbarga Karnataka in the 1993, M.Tech. degree from the AAIDU Allahabad in 2005, and the Ph.D. degree from the University of Singhania, Rajasthan India in 2012. From 1993 to 2010 he worked as a Lecturer, Senior Lecturer and Assistant professor and HOD Dept. of CSE \& ISE at SLN College of Engineering, Raichur Karnataka. From 2010 to June 2019 he worked as a an Associate Professor and HOD in the Department of Computer Science and Engineering at Government Engineering College Raichur, Karnataka. He

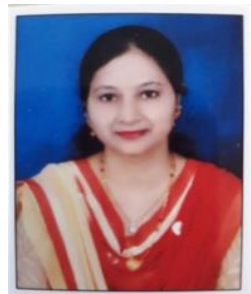
is currently Working as Principal Government Engineering College, Gangavathi, Karnataka from July 2019. His research interests are in Image Processing and Computer Network.

Vishwanath $\mathbf{P}$ completed his M.E from PDA college of Engineering, Gulbarga University, Gulbarga (Karnataka) in the year 2003 and completed his $\mathrm{PhD}$ in the year 2017 from NIMS University, Jaipur (Rajasthan). Presently working as Associate Professor in the department of "Electronics and Communication Engineering" since 1998.His area of interest are Image Processing and Embedded System. He has publishes four papers in international journals and presented one paper in international conference.

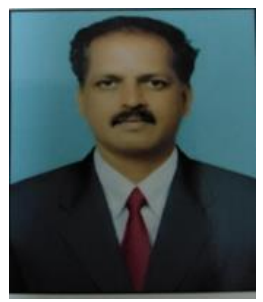

